DOE/NV/25946--164

\title{
LARGE FORMAT X-RAY PINHOLE CAMERA
}

\author{
Ming $\mathrm{Wu}^{1}$, Aric Tibbitts, ${ }^{1}$ Nathan $\mathrm{Joseph}^{1}$, and \\ Gordon A. Chandler ${ }^{2}$ \\ ${ }^{1}$ National Security Technologies, LLC, Los Alamos, \\ NM 87544, USA \\ ${ }^{2}$ Sandia National Laboratories, Albuquerque, \\ NM 87185, USA
}

National Security Technologies, LLC, has successfully implemented many scientific and engineering innovations in the new Large-Format Pinhole Camera (LFPHC), which have dramatically increased the detection sensitivity and reliability of the camera in exotic locations, such as the Sandia National Laboratories Z-facility. Quality improvements of the LFPHC have been demonstrated in its fielding at $\mathrm{Z}$, where high-quality images were recorded. A major improvement was the development of a new, user-friendly LFPHC camera back that would tolerate high radiation, electromagnetic interference, and mechanical shock. Key modifications resulted in improved detection sensitivity, spatial resolution, uniformity along the microchannel plate strip, and stability of the interframe timing and delay. Design considerations and improvements will be discussed.

This work was done by National Security Technologies, LLC, under Contract No. DE-AC52-06NA25946 with the U.S. Department of Energy. 